\title{
Polymer surface properties control the function of heavy meromyosin in dynamic nanodevices
}

\author{
Kristi L. Hanson ${ }^{1 *}$, Florin Fulga ${ }^{2 *}$, Serban Dobroiu ${ }^{2}$, Gerardin Solana ${ }^{1}$, Ondrej Kaspar ${ }^{3}$, Viola \\ Tokarova $^{3}$, Dan V. Nicolau ${ }^{1,2,3}$ \\ ${ }^{1}$ Industrial Research Institute Swinburne, Swinburne University of Technology, Hawthorn, \\ Victoria, 3122, Australia \\ ${ }^{2}$ Department of Electrical Engineering and Electronics, The University of Liverpool, Liverpool, \\ L69 3GJ, United Kingdom \\ ${ }^{3}$ Department of Bioengineering, McGill University, Montreal, Quebec, Canada H3A 0C3
}

*KLH and FF contributed equally.

\begin{abstract}
The actin-myosin system, responsible for muscle contraction, is also the force-generating element in dynamic nanodevices operating with surface-immobilized motor proteins. These devices require materials that are amenable to micro- and nano-fabrication, but also preserve the bioactivity of molecular motors. The complexity of the protein-surface systems is greatly amplified by those of the polymer-fluid interface; and of the structure and function of molecular motors, making the study of these interactions critical to the success of molecular motor-based nanodevices. We measured the density of the adsorbed motor protein (heavy meromyosin, HMM) using quartz crystal microbalance; and motor bioactivity with ATPase assay, on a set of model surfaces, i.e., nitrocellulose, polystyrene, poly(methyl methacrylate), and poly(butyl methacrylate), poly(tert-butyl methacrylate). A higher hydrophobicity of the adsorbing material translates in a higher total number of HMM molecules per unit area, but also in a lower uptake of water, and a lower ratio of active per total HMM molecules per unit area. We also measured the motility characteristics of actin filaments on the model surfaces, i.e., velocity, smoothness and deflection of movement, determined via in vitro motility assays. The filament velocities were found to be controlled by the relative number of active HMM per total motors, rather than their absolute surface density. The study allowed the formulation of the general engineering principles for the selection of polymeric materials for the manufacturing of dynamic nanodevices using protein molecular motors.
\end{abstract}




\title{
Polymer surface properties control the function of heavy meromyosin in dynamic nanodevices
}

\author{
Kristi L. Hanson, Florin Fulga, Serban Dobroiu, Gerardin Solana, Ondrej Kaspar, Viola \\ Tokarova, Dan V. Nicolau
}

\section{Introduction}

Mechanical work in biological nanosystems is performed by a variety of force-generating protein motors, such as myosins, kinesins and dyneins,(Spudich 2011; Vale 2003; Veigel and Schmidt 2011) the former being responsible for muscle contraction.(Huxley and Niedergerke 1954; Huxley and Hanson 1954) In the "gliding geometry" motility assay developed in the late 1980s, whole myosin molecules,(Kron and Spudich 1986; Uyeda et al. 1991) or the part of the mechano-enzyme containing its working arms, e.g., heavy meromyosin (HMM), (Uyeda et al. 1991) or even the end of an arm, i.e., the S1 unit,(Toyoshima et al. 1987; Uyeda et al. 1991) are adsorbed on a surface. Provided that the upper solution contains sufficient adenosine triphosphate (ATP), the fluorescently-labelled actin filaments will be propelled by the surfacebound motors, sliding randomly on the surface, thus allowing the easy observation and quantification of motility characteristics using simple optical fluorescence microscopy setups and imaging software.

While the "gliding geometry" motility assay has been used extensively in fundamental studies of molecular motor function(Holzbaur and Goldman 2010), from an applications perspective, their planar architecture and the ability of motor proteins to transport nano-scale cargo at speeds that are orders of magnitude higher than those associated with molecular diffusion,(Nitta and Hess 2005) are very attractive features for dynamic nanodevices(Bakewell and Nicolau 2007; Fulga et al. 2009; Kinbara and Aida 2005) Consequently, proof-of-concept motor-powered nanodevices have been proposed for biosensing,(Agarwal et al. 2009; Martinez-Neira et al. 2005; Van Zalinge et al. 2012) biodiagnostics,(Fischer et al. 2009; Korten et al. 2010) transport at nano-,(Bull et al. 2005) and micro-scale,(Limberis and Stewart 2000), microfluidic pumping(Bull et al. 2005) and recently biocomputation.(Nicolau et al. 2016)

Because the manufacturing of these devices must use materials that are both suitable for micro/nanofabrication, and also preserve the motor bioactivity, various materials have been assessed, e.g., methacrylate polymers,(Nicolau et al. 1999; Riveline et al. 1998; Suzuki et al. 1997) polyurethane,(Clemmens et al. 2003a) plasma polymerized poly(ethylene oxide),(Clemmens et al. 2003b) polyelectrolytes,(Jaber et al. 2003) commercial photoresists,(Bunk et al. 2003a; Bunk et al. 2003b; Clemmens et al. 2004; Hiratsuka et al. 2001; Moorjani et al. 2003) and silane-functionalized surfaces.(Bunk et al. 2005; Sundberg et al. 2003) Despite this rather large amount of empirical information, as well as several studies focused on the fundamentals of the motor protein-surface interactions,(Albet-Torres et al. 2007a; Katira et al. 2007; Katira et al. 2009; Van Zalinge et al. 2012) there are still many uncertainties regarding the impact of surfaces on motor function, in particular regarding polymers, which are the preferred materials for inexpensive devices, due to the coupled complexities of the polymer and the protein systems. 
To this end, to progress on the selection of materials for future dynamic nanodevices using actinmyosin system, we studied the relationship between the physico-chemical properties polymeric surfaces, in particular their hydrophobicity and polymer network structure, on one side; and the surface density of molecular motors and the preservation of their motility, on the other.

\section{Materials and Methods}

Polymer surface coating. Superclean nitrocellulose (NC) was purchased from Ernest F. Fullam, Inc. (Latham, NY). Polystyrene (PS), poly(methyl methacrylate) (PMMA), poly(butyl methacrylate) (PBMA), poly(tert-butyl methacrylate) (PtBMA) and hexamethyldisilazane (HMDS) were purchased from Aldrich Chemicals. The selection of polymeric surfaces aims to reach a reasonably large range of properties related to motility assays and related devices: (i) nitrocellulose is the standard polymer for motility assays, but unfit for the fabrication of devices due to its flammability; (ii) polystyrene is the material of choice for the plastic utensils in molecular biology and biochemistry, but is rarely used for motility assays; (iii) PMMA is the material of choice for polymer-based microfluidics devices, and has been also used with good results for motility assays; (iv) it would be useful to compare PMMA with other more hydrophobic acrylates, but with very different properties related to water uptake, i.e., high, and low Tg, for PtBuMA and PBMA, respectively. Finally, while silane polymers, e.g., poly(di methyl siloxane), PDMS, are used indeed for microfluidics devices, the release of mono-/oligomers is extremely toxic for motility assays.

The chemical structures for all model surfaces are presented in Figure 1.

\section{[Figure 1]}

Glass coverslips were cleaned by sonication in $70 \%$ ethanol, dried in a stream of $\mathrm{N}_{2}$, primed with HMDS, spin-coated with one of the polymer solutions, i.e., NC (1\% w:v in amyl acetate), PS (2.5\% w:v in propylene glycol monomethyl ether acetate, PGMEA), PMMA (2\% w:v PGMEA), PBMA (1\% w:v toluene), or PtBMA (2\% w:v in PGMEA) at $3600 \mathrm{rpm}$, then soft-baked at $85^{\circ} \mathrm{C}$ for three hours. The concentrations of solvents in the polymer solutions have been optimised to obtain a viscosity that leads to a smooth film during the spin coating.

The hydrophobicities of the polymer-coated surfaces were determined by contact-angle measurements using deionized water $(\mathrm{R}>18.2 \mathrm{M} \Omega)$ and Krüss contact-anglemeter (DSA10Mk2). The reported values are averages of ten different readings for each surface.

The measurement of the viscoelastic properties of the polymers in thin films used an advanced commercial quartz microbalance (QCM) system (QCM-Z500, from KSV Instruments). This system allowed the measurement of the impedance spectrum, thus providing both the frequency and the bandwidth, addressing up to $11^{\text {th }}$ harmonics. The measurements have been performed sequentially, in a step-wise manner, i.e., first on the bare dry polymer surfaces, then on surfaces interfaced with the buffer solution. A complete description of the QCM equipment, measurement protocols and associated theoretical background is presented in the Supplementary Information Section.

Protein preparation. Myosin and G-actin were extracted and purified from rabbit skeletal back muscle using previously reported methods.(Barden and Dosremedios 1984; Carsten and Mommaerts 1963; Margossian and Lowey 1982; Spudich and Watt 1971) HMM was prepared by proteolytic cleavage of myosin $\left(20 \mathrm{mg} \mathrm{mL}^{-1}\right.$ in $\left.0.5 \mathrm{M} \mathrm{KCl}, 50 \mathrm{mM} \mathrm{KPO} 4 \mathrm{pH} 6.5\right)$ with 0.07 $\mathrm{mg} \mathrm{mL}^{-1} \alpha$-chymotrypsin. Prior to use in motility studies, G-actin was dissolved in $1 \mathrm{mM}$ DTT 
to a concentration of $2 \mathrm{mg} \mathrm{mL}^{-1}$ and clarified by centrifugation (40,000 rpm for $60 \mathrm{~min}$ ). F-actin was simultaneously polymerized and fluorescently labeled by incubation of equal molar quantities of G-actin and AlexaFluor 488 phalloidin (Molecular Probes) in $4 \mathrm{mM}$ imidazole $\mathrm{pH}$ 7, $2 \mathrm{mM} \mathrm{MgCl} 2,0.1 \mathrm{mM}$ EGTA, $3 \mathrm{mM} \mathrm{NaN}_{3}, 1 \mathrm{mM}$ DTT.

Motility assays. Actomyosin motility assay procedures were modified from Sellers et al.(Sellers et al. 1993) The motility flow cells were constructed by sealing two parallel edges of a polymercoated coverslip to a standard glass microscope slide using double-sided tape (approximately 100 $\mu \mathrm{m}$ thickness) as a spacer. HMM was diluted to $0.1 \mathrm{mg} \mathrm{mL}^{-1}$ in $10 \mathrm{mM}$ MOPS pH 7.2, $20 \mathrm{mM}$ $\mathrm{KCl}, 5 \mathrm{mM} \mathrm{MgCl}$, $0.1 \mathrm{mM}$ EGTA, $10 \mathrm{mM}$ DTT (Buffer A, which is classically used for myosin-based motility assays, e.g., Sellers et al., 1993), introduced into the flow cell and allowed to attach on the surface for 2 minutes. The cell was then flushed sequentially with the following (all in Buffer A): (i) $1 \mathrm{mg} \mathrm{mL}^{-1} \mathrm{BSA}$, (ii) $5 \mu \mathrm{M}$ unlabelled F-actin, (iii) $1.5 \mathrm{mM} \mathrm{MgATP}$, and (iv) $5 \mathrm{nM}$ labelled actin. The motility was initiated by the addition of $1 \mathrm{mM} \mathrm{MgATP}$ in Buffer A. A photobleaching inhibition solution, consisting of $2.5 \mathrm{mg} \mathrm{mL}^{-1}$ glucose, $0.1 \mathrm{mg} \mathrm{mL}^{-1}$ glucose oxidase (Sigma G-7016) and $0.02 \mathrm{mg} \mathrm{mL}^{-1}$ catalase (Sigma C-100), was also added.

Motility Data Analysis. The motility was observed at room temperature $\left(23-25^{\circ} \mathrm{C}\right)$ on an inverted microscope (Olympus IX71) using a PlanApo 100x oil objective (Olympus), epifluorescence optics (FITC filter set), and mercury light source. Images were recorded every $150 \mathrm{~ms}$ for $60 \mathrm{sec}$ using a Coolview FDI high-resolution camera (Photonics Science Ltd.) controlled by Image-Pro Plus software (Ver. 5.0, Media Cybernetics). The manual object tracking features of Image-Pro Plus were used to determine the coordinates of actin filament heads in consecutive frames, from which the motility statistics were derived.

Replicate trials for each surface were conducted, with all filaments within one quadrant (representing a $44 \times 33 \mu \mathrm{m}^{2}$ area) of the field of view being evaluated, resulting in 20 to 32 filaments being analysed for each surface.

The step velocity was calculated as the distance travelled by the filament head divided by the time between sampled frames. The step velocity variation was calculated as the difference between two consecutive velocities. The deflection angle was calculated for each combination of three consecutive points as the difference between (i) the direction of filament head movement between two consecutive points; and (ii) the direction of filament head movement between the next two consecutive points. The absolute value of the directional difference was calculated, such that the angle of the directional change was always less than $180^{\circ}$.

Measurement of the surface density of adsorbed HMM. The same advanced QCM system (QCMZ500, KSV Instruments) was used to measure the amount of adsorbed protein mass, and to assess the dynamics of viscoelastic properties of the thin films comprising proteins on, or embedded in polymers. The measurements used similar protocols as for the measurement of viscoelastic properties of the dry, and buffer-soaked polymer films. A complete description of the QCM equipment, measurement protocols and associated theoretical background is presented in the Supplementary Information Section.

Measurement of HMM activity. The amount of active HMM bound to polymer-coated coverslips was estimated by comparing $\mathrm{NH}_{4}$-EDTA ATPase activities in flow cells with those of known amounts of $\mathrm{HMM}$ in solution (all at $25^{\circ} \mathrm{C}$ ). In solution, ATPase activities were determined colorimetrically based on the rate of inorganic phosphate release(Taussky and Shorr 1953) by known concentrations of HMM in ATPase buffer $(25 \mathrm{mM}$ imidazole hydrochloride, $0.2 \mathrm{M}$ $\mathrm{NH}_{4} \mathrm{Cl}, 9 \mathrm{mM}$ EDTA, $\mathrm{pH}$ 7.4). The reaction was started by addition of $5 \mathrm{mM}$ ATP and stopped 
by the addition of an equal volume of Taussky-Shorr reagent (1\% [w:v] ammonium molybdate, $5 \%$ [w:v] ferrous sulphate heptahydrate in $0.5 \mathrm{M} \mathrm{H}_{2} \mathrm{SO}_{4}$ ). ATPase activities of surface immobilized HMM were determined in flow cells of HMM under standard motility assay conditions (see further). After equilibration of HMM in Buffer A, the flow cell was rinsed with ATPase buffer and the reaction started by the introduction of $5 \mathrm{mM}$ ATP in ATPase buffer. After set reaction times, chosen at 5, and 10 mins, respectively, the flow cell contents were removed by perfusion with 4 volumes $(\sim 200 \mu \mathrm{L})$ ATPase buffer (no ATP), the total volume was brought to 1 $\mathrm{mL}$, and the reaction stopped by the addition of an equal volume of Taussky-Shorr reagent. In order to ensure a full and stable consumption of ATP, we worked with HMM concentrations that are double than those reported elsewhere(Guo and Guilford 2004) as limiting the rate of ATP consumption.

\section{Results and Discussion}

Complex relationship between the surface properties and motility characteristics. The characteristics of the motility of cytoskeletal filaments on surface immobilized motor proteins in in vitro motility assays is a function of (i) the characteristics intrinsic of the motor system, e.g., ATP cycling time, power stroke length, strong binding time,(Fulga et al. 2009; Veigel and Schmidt 2011) and (ii) the assay-related characteristics, e.g., the density of motor proteins on the surface, their activity, and ATP concentration.(Albet-Torres et al. 2007a; Nicolau et al. 2007a) Aside of the above elements, the characteristics of the adsorbing surface in controlling the surface density of motors and their surface-controlled bioactivity, but the measurement of both these parameters has been very rarely reported. For instance, early experimental and theoretical studies (Harris and Warshaw 1993; Riveline et al. 1998; Uyeda et al. 1990) examined the correlation between the velocity of actin filaments as a function of the surface density of active motors (and their intrinsic parameters), as estimated from ATPase assays. Moreover, the analysis used a single type of surface, most frequently nitrocellulose, but overlooking the surface density of denaturated, inactive motor molecules. Alternatively, the impact of the surface properties on the motor bioactivity was indirectly estimated by comparing the average actin filaments velocity on motor protein immobilized on various surfaces.(Bunk et al. 2003a; Jaber et al. 2003; Nicolau et al. 2007b; Nicolau et al. 1999; Sundberg et al. 2003) Finally, the amount of surfaceimmobilized motors was measured using QCM,(Albet-Torres et al. 2010; Albet-Torres et al. 2007b; Van Zalinge et al. 2012) but the concomitant report of the still active motors, estimated using ATPase assay, has been reported only once,(Albet-Torres et al. 2007a) and on nonpolymeric surfaces. Interestingly, it has been observed that the average velocity of actin filaments increases with the hydrophobicity of the surfaces that immobilize myosin, if this surface is flat and rigid, e.g., silanised glass.(Sundberg et al. 2006) However, in a clear sign that other factors are at play in determining the bioactivity of the motors, the average velocity of actin filaments on motor-functionalised polymers degreases with the increase of the surface hydrophobicity beyond a certain threshold,(Nicolau et al. 2007b) Taking into account this contradictory information, the goal of the present study is to evaluate the impact of both polymer hydrophobicity and its viscoelastic properties on myosin density, activity, and motility of the actin filaments, to be able to appropriately select polymeric materials for molecular motors-based nanodevices.

Polymer properties control the amount of adsorbed HMM. Several studies(Fawcett et al. 1998; Hook et al. 2001; Hook et al. 2002; Rickert et al. 1997) demonstrated that protein layers 
adsorbed on surface incorporate substantial amounts of water. For instance, QCM measurements have demonstrated (Caruso et al. 1995; Geddes et al. 1994; Hook et al. 2002; Rickert et al. 1997) that, due to the additional mass of incorporated water, the frequency change following the protein adsorption in a liquid environment is 4x (Caruso et al. 1995; Geddes et al. 1994; Hook et al. 2002; Rickert et al. 1997) to 10x (Albet-Torres et al. 2007a; Hook et al. 2001) times higher than those in air. The QCM measurements of adsorbed masses on rigid layers is nearly trivial, i.e., fully described by the classical Sauerbrey equation,(Sauerbrey 1959) but data interpretation for non-rigid layers is considerably more difficult because of the coupling of viscoelastic effects for soft, gel-like layers. However, the capacity of QCM methodology to measure both the adsorbed mass, as well as the viscoelastic properties of the protein layers, has been used to estimate these properties separately, e.g., the amount of motor protein on rigid surfaces, (AlbetTorres et al. 2007a; Hanson et al. 2006) and the conformation of the protein motor layer as transducing the motility state in non-optical signal,(Van Zalinge et al. 2012) respectively. Therefore, the measurements of (i) the absorbed water in the polymer layers, followed by (ii) the measurement of the adsorbed motors on the polymer surfaces, and finally (iii) the measurements of the absorbed water in the protein layers, is likely to progress the understanding of the behaviour of motor proteins immobilized on, or into polymeric layers.

The water uptake in the polymer film, i.e., before immobilization of HMM, is generally lower for more hydrophobic polymers (Supplementary Information, Figure SI1). While this relationship is generally expected, NC absorbs an unusual high amount of water, i.e., nearly $30 \%$ more than the next water-absorbing polymer (PMMA), with which it clusters as higher water-adsorbing polymers. This suggests that NC, and to a lesser extent PMMA, form thick gel-like layers in/on which the HMM molecules are immobilized. Equally expected, the water uptake is lower for polymers having higher glass transition (Tg), with the notable exception of PBMA (Supplementary Information, Figure SI2). For glassy polymers, i.e., all tested less PBMA, a high $\mathrm{Tg}$ is the result of a higher stiffness of the polymer chains, which will decrease the rate of water diffusion. Conversely, for the rubbery PBMA, the much more flexible polymer network amplifies the impact of its hydrophobicity, thus leading to far less water uptake than it would be otherwise expected for its contact angle. Finally, the water uptake appears to increase, for glassy polymers, with their density (Supplementary Information, Figure SI3). This apparently counterintuitive trend can be understood by the larger content of hydrophilic groups, i.e., hydroxyl groups for $\mathrm{NC}$, carboxyl groups for PMMA, which will result in stronger hydrogen bonding, and subsequently more compact polymeric networks and higher density.

A higher polymer surface hydrophobicity results in a higher amount of total adsorbed HMM (Figure 2a). This strong relationship between surface hydrophobicity and the mass of adsorbed protein has been explained before in general terms,(Ostuni et al. 2003; Vasina et al. 2009) as well as specifically regarding HMM adsorption.(Albet-Torres et al. 2007a; Nicolau et al. 2007b; Sundberg et al. 2003; Van Zalinge et al. 2012) Interestingly, NC presents the lowest amount of adsorbed HMM, despite the fact that it is the standard surface used in motility assays studies. Conversely, PS, which has not reported as being used in motility studies, although it is commonly used in assays using surface-immobilized proteins, presents the highest adsorbed amount of HMM. Importantly for the understanding of the control of motility characteristics by polymer properties, a higher water uptake in the polymer, which is largely in an inverse relationship with the polymer hydrophobicity, albeit not in an univoque manner, results in a lower amount of HMM adsorbed on, or rather absorbed in, the polymer layer (Figure $2 b$ ). 
Finally, the uptake of water in HMM layer correlates well with the water uptake in the polymer layer (Supplementary Information, Figure SI4).

\section{[Figure 2]}

Structure of the polymer-HMM layer. The estimation of the mass of surface-adsorbed HMM allows the calculation of the total surface density of HMM molecules (Table 1). The overall contribution to the acoustic impedance is calculated by matrix multiplication, starting from the top layer, the HMM solution. The results of these calculations are presented in the Supplementary Information section (Table SI2). One observation is that the density of the protein layer for all surfaces is approximately the same, i.e., $\sim 1090 \mathrm{~kg} \mathrm{~m}^{-3}$, which is close to the values reported elsewhere,(Hook et al. 2002)) e.g., $1040 \mathrm{~kg} \mathrm{~m}^{-3}$. However, there are notable differences in the thickness of the protein layers, which suggests different conformations of the motor protein on different surfaces, and possibly its surface-bound site. The values obtained for the total mass adsorbed and the number of heads, i.e., $280-540 \mathrm{ng} / \mathrm{cm}^{2}$, which depend on the surface properties (higher on hydrophobic surfaces), are similar with those reported elsewhere,(Harada 1990) i.e., $350 \mathrm{ng} / \mathrm{cm}^{2}$ for myosin on glass.

\section{[Table 1]}

A vertically-structured, multi-layer model, comprising, from bottom up, [solid substrate]-[stiff polymer]-[polymer gel]-[HMM layer], has been recently proposed,(Van Zalinge et al. 2012) but the present experiments allow a more precise description, as follows:

(i) At the bottom of the multilayer, the basal surface supports polymer layer of 500-750 nm, which contains various amounts of water, as described in the previous sections.

(ii) This polymer layer supports a HMM-rich layer, which also contains water. This HMM layer comprises two sub-layers with different densities and dynamical moduli, thus contributing differently to the acoustic impedance. A bottom sub-layer, several nm's thick, which due to its reduced thickness can be treated within the Sauerbrey approximation, is covered by a top sublayer with a thickness of $\sim 15-35 \mathrm{~nm}$, rich in water, comprising one or both $\mathrm{S} 1$ heads, and which has the characteristics of a gel, thus modelled as a viscoelastic layer.

(iii) The constitutive element of the polymer-protein layer, i.e., HMM molecule, is 80-90 nm long, including the S1 heads, which are $19 \mathrm{~nm}$ long with a diameter of 2-3 nm. These geometrical considerations, coupled with the large amount of water trapped in the protein layer suggest an arrangement of HMM molecules with the head groups tightly packed and the long axes of the motors perpendicular to the surface, as proposed before.(Albet-Torres et al. 2007a)

The [solid substrate]-[stiff polymer]-[polymer gel]-[HMM layer] model described above is schematically presented in Figure 3.

\section{[Figure 3]}

Polymer properties control the amount of active HMM. The immediate observation when comparing the density of QCM-measured, total HMM molecules (Figure 2a) and ATPasemeasured, active HMM molecules (Figure 4a), is that the surface hydrophobicity has a contradictory effect on these two parameters. Indeed, as proposed above and elsewhere,(Nicolau et al. 2007b) the surfaces with higher hydrophobicity induce the adsorption of a higher total number of HMM molecules, but also a lower number of active HMM molecules. This is not surprising, as hydrophobic surfaces will almost always induce a larger adsorption of proteins.(Ostuni et al. 2003; Vasina et al. 2009). Moreover, the alternative-to-hydrophobicity 
driving force for protein adsorption, i.e., electrostatic interactions, will be more prevalent on hard, molecularly flat surfaces, e.g., silicon-based materials(Albet-Torres et al. 2007a), as the short-range hydrophobic interactions will be prevalent due to the brush-like nature of polymeric surfaces. Also, the hydrophobic interactions between the polymeric surfaces and motor proteins will result in the expulsion of water starting from the hydrophobic core outwards the protein, thus inducing its denaturation(Ostuni et al. 2003). The overall result of this 'tug-of-war' between larger total HMM molecules, but also lower active HMM molecules, is a decrease of the ratio of active vs. total HMM molecular density with surface hydrophobicity. An increase of the water uptake in the polymer (in negative correlation with polymer hydrophobicity), results in an increase of both active HMM molecules, and importantly the ratio between the active and total number of HMM molecules (Figure 4b).

\section{[Figure 4]}

Polymer properties control the motility of actin filaments. The various amounts of total HMM molecules adsorbed on various polymer surfaces, and in particular the impact of the properties of respective polymers on the preservation of bioactivity of motor proteins, result in variations of the characteristics of the motility of actin filaments sliding on HMM-functionalised surfaces (Figure 5, also Table 1). The analysis of the characteristics of the motility of actin filaments on surfaces shows that only NC induces a rapid and smooth movement, indicated by the monomodal distribution of velocities, skewed towards higher values, and monotonous distribution of deflection angles skewed towards 0 degrees. All other surfaces present bimodal distributions of velocities, which could be related to denaturated HMM stalling the motility, or a population of partially-denaturated HMM working with a single arm, which will propel actin filaments at lower velocities.(Toyoshima et al. 1987) Also all surfaces other than NC (and possibly PMMA) induce a 'stuttered' motion of the actin filaments as evidenced by the broader band of frequency on both sides of 0 values for acceleration (an acceleration means perfectly smooth movement), and large proportion of non-zero deflection angles. This behavior can be understood by the higher proportion of non-active HMM molecules, which will stall or brake the motion of actin filaments.

A clear, but initially counterintuitive trend, that is, of the decreasing velocity of actin filaments with the increase of the total HMM molecular density can be observed in Figure 6a. Rather expectedly, the increase of the active HMM leads to an increase of the actin filament velocity (Figure 6b). Finally, a stronger positive correlation exists between filament velocity and the proportion of active to total heads (Figure 6c). Of particular note in these results is that NC, which is typically considered the 'gold standard' for actomyosin motility, presents by far the highest proportion of active per total HMM (also Table 1). The relationship in Figure 6c is positive, consistent with previously described models,(Cuda et al. 1997; Tawada and Sekimoto 1991; Warshaw et al. 1990) which assume that the in vitro actomyosin velocity is the result of a balance between myosin cross-bridges in the productive force-generating state and external load imposed by non-productive cyclical actin-myosin interactions.

Selection of materials for dynamic nanodevices using molecular motors. In the view of the complex relationship between the properties of the surfaces immobilising protein molecular motors, in particular myosin, as described above, the general guidelines for the selection of the polymeric materials to be employed in the manufacturing of dynamic nanodevices can be summarised as follows: 
- Key performance criteria for motility. Different dynamic nanodevices using protein molecular motors, would require different key performance features, depending on their intended application. For instance, diagnostic devices can capitalise on the much higher rate of interaction between the target and the probe molecules than that driven by diffusion, as in classical assays, e.g., ELISA.(Nitta and Hess 2005) For these devices, which transduce the biomolecular recognition into change of motility,(Korten et al. 2010; Kumar et al. 2016; Martinez-Neira et al. 2005; Van Zalinge et al. 2012) and which will interact with various biological fluids,(Korten et al. 2013) the maintenance of motility is paramount. On the other hand, in other applications, such as diagnostic devices using molecular motors to transport and concentrate the target molecules in selected locations(Kumar et al. 2016; Lard et al. 2013); or biocomputation devices,(Nicolau et al. 2016) a high velocity of the cytoskeletal filaments could become more important. As a general rule, however, the maintenance of the motility, ensured by the presence of a large number of active motors, and/or the preservation of their bioactivity is critical for any successful implementation of dynamic nanodevices using protein molecular motors.

- Surface hydrophobicity. Materials with a high surface hydrophobicity will induce a high total amount of adsorbed motors, which would be expected to translate in a higher density of active motors. However, for polymeric materials, it was demonstrated here that a higher hydrophobicity translates in high rates of denaturation, which greatly overshadow the benefits of higher overall total density of motors. Furthermore, denaturated motors could have a deleterious effect on motility. Consequently, for polymeric surfaces, a decreased hydrophobicity is likely to be beneficial for the motility of cytoskeletal filaments. It appears that this inverse relationship between surface hydrophobicity and preservation of the bioactivity of the motors is specific to polymeric materials, as it has been demonstrated that of very flat, very rigid surfaces, e.g., silanised silicon oxide,(Albet-Torres et al. 2007a) a higher hydrophobicity results in higher amounts of total and active motors. While these rigid surfaces appear to be address the key performance criteria regarding motility, polymeric materials offer important advantages regarding fabrication, cost and disposability.(Becker and Gärtner 2000; Sollier et al. 2011)

- Water uptake in polymers. It appears that the preservation of the activity of the motors is not only due to a low hydrophobicity of the material, but also by the capacity of the polymer to form gel-like films via water uptake. Indeed, while NC is not the most hydrophilic polymer studied here, its very high capacity for water uptake, even comparing with the nearest candidate (PMMA) makes it the best material regarding the preservation of motor activity.

- New materials tailored for dynamic nanodevices. The present study demonstrated the physico-chemical reasons why $\mathrm{NC}$ is the 'gold standard' of motility assays, i.e., moderate hydrophobicity, and high level of water uptake. However, for microfluidics devices, NC is far from being an optimal material for their fabrication and operation, e.g., due to its inflammability. The key performance parameters demonstrated here, i.e., moderate hydrophobicity, and higher capacity for water uptake, as well as other, e.g., optical transparency, could be the leads for the search of other materials for dynamic nanodevices. For instance, cyclo-olefin copolymers (COC) have been demonstrated to be appropriate for micro-manufacturing,(Mair et al. 2006) and also to have the appropriate physico-chemical properties for biomolecule immobilisation.(Nicolau et al. 2005) 


\section{Conclusions}

We have investigated the density and bioactivity of HMM on a variety of polymer surfaces, and related the results to in vitro actomyosin motility velocities. The results show that the proportion of active per total HMM heads, rather than the surface density of active HMM, controls the actin filament sliding velocities. We have thus shown that the mechanism by which surfaces modulate actomyosin motility in vitro is complex, such that HMM molecules adsorbed to a surface in a perturbed conformation or ineffective orientation appear to play a significant role in decreasing filament sliding velocities. The results have application to the fundamental understanding of actin and myosin interactions, and to the future engineering of surfaces for molecular motor based nano-devices.

\section{Acknowledgements}

This work has been supported by grants from the Defense Advanced Research Projects Agency, under grant agreements N66001-00-1-8952 and N66001-03-1-8913; the Australian Research Council (Discovery project DP0208754); European Union Seventh Framework Programme (FP7/2007-2011) under grant agreement number 228971 (MONAD); and Natural Science and Engineering Research Council (NSERC, Canada), grant RGPIN-2016-05019. The authors wish to thank Dr. Murat Kekic and Prof. Cristobal dos Remedios from the University of Sydney, and Dr. Jenny Aveyard from the University of Liverpool, for myosin preparation; and to Dr. Harm van Zalinge for assistance with QCM measurements.

Contributions of the authors. KLH run the motility and ATPase assays. GS collected the motility data. SD run the QCM experiments. KLH, GS, FF, SD, OK, VT and DVN analysed the data. $\mathrm{KLH}, \mathrm{FF}, \mathrm{OK}$, and DVN wrote the paper. 


\section{References}

Agarwal, A., Katira, P., Hess, H., 2009. Millisecond Curing Time of a Molecular Adhesive Causes Velocity-Dependent Cargo-Loading of Molecular Shuttles. Nano Letters 9(3), 11701175.

Albet-Torres, N., Gunnarsson, A., Persson, M., Balaz, M., Höök, F., Månsson, A., 2010. Molecular motors on lipid bilayers and silicon dioxide: Different driving forces for adsorption. Soft Matter 6(14), 3211-3219.

Albet-Torres, N., O'Mahony, J., Charlton, C., Balaz, M., Lisboa, P., Aastrup, T., Mansson, A., Nicholls, I.A., 2007a. Mode of heavy meromyosin adsorption and motor function correlated with surface hydrophobicity and charge. Langmuir 23, 11147-11156.

Albet-Torres, N., O'Mahony, J., Charlton, C., Balaz, M., Lisboa, P., Aastrup, T., Månsson, A., Nicholls, I.A., 2007b. Mode of heavy meromyosin adsorption and motor function correlated with surface hydrophobicity and charge. Langmuir 23(22), 11147-11156.

Bakewell, D.J.G., Nicolau, D.V., 2007. Protein linear molecular motor-powered nanodevices. Australian Journal of Chemistry 60(5), 314-332.

Barden, J.A., Dosremedios, C.G., 1984. The environment of teh high-affinity cation binding site on actin and the separation between cation and ATP sites as revealed by protein NMR and fluorescence spectroscopy. Journal of Biochemistry 96(3), 913-921.

Becker, H., Gärtner, C., 2000. Polymer microfabrication methods for microfluidic analytical applications. Electrophoresis 21(1), 12-26.

Bull, J.L., Hunt, A.J., Meyhofer, E., 2005. A theoretical model of a molecular-motor-powered pump. Biomedical Microdevices 7(1), 21-33.

Bunk, R., Klinth, J., Montelius, L., Nicholls, I.A., Omling, P., Tagerud, S., Mansson, A., 2003a. Actomyosin motility on nanostructured surfaces. Biochemical and Biophysical Research Communications 301(3), 783-788.

Bunk, R., Klinth, J., Rosengren, J., Nicholls, I., Tagerud, S., Omling, P., Mansson, A., Montelius, L., 2003b. Towards a 'nano-traffic' system powered by molecular motors. Microelectronic Engineering 67-8, 899-904.

Bunk, R., Sundberg, M., Mansson, A., Nicholls, I.A., Omling, P., Tagerud, S., Montelius, L., 2005. Guiding motor-propelled molecules with nanoscale precision through silanized bi-channel structures. Nanotechnology 16(6), 710-717.

Carsten, M.E., Mommaerts, W.F.H., 1963. A study of actin by means of starch gel electrophoresis. Biochemistry 2(1), 28-\&.

Caruso, F., Serizawa, T., Furlong, D.N., Okahata, Y., 1995. Quartz-crystal microbalance and surface-plasmon resonance study of surfacctant adsorption onto gold and chromium-oxide surfaces. Langmuir 11(5), 1546-1552.

Clemmens, J., Hess, H., Doot, R., Matzke, C.M., Bachand, G.D., Vogel, V., 2004. Motor-protein "roundabouts": Microtubules moving on kinesin-coated tracks through engineered networks. Lab on a Chip 4(2), 83-86.

Clemmens, J., Hess, H., Howard, J., Vogel, V., 2003a. Analysis of microtubule guidance in open microfabricated channels coated with the motor protein kinesin. Langmuir 19(5), 1738-1744.

Clemmens, J., Hess, H., Lipscomb, R., Hanein, Y., Bohringer, K.F., Matzke, C.M., Bachand, G.D., Bunker, B.C., Vogel, V., 2003b. Mechanisms of microtubule guiding on microfabricated kinesin-coated surfaces: Chemical and topographic surface patterns. Langmuir 19(26), 1096710974. 
Cuda, G., Pate, E., Cooke, R., Sellers, J.R., 1997. In vitro actin filament sliding velocities produced by mixtures of different types of myosin. Biophysical Journal 72(4), 1767-1779.

Fawcett, N.C., Craven, R.D., Zhang, P., Evans, J.A., 1998. QCM Response to Solvated, Tethered Macromolecules. Analytical Chemistry 70, 2876-2880.

Fischer, T., Agarwal, A., Hess, H., 2009. A smart dust biosensor powered by kinesin motors. Nature Nanotechnology 4(3), 162-166.

Fulga, F., Nicolau, J., D. V., Nicolau, D.V., 2009. Models of protein linear molecular motors for dynamic nanodevices. Integrative Biology 1(2), 150-169.

Geddes, N.J., Paschinger, E.M., Furlong, D.N., Ebara, Y., Okahata, Y., Than, K.A., Edgar, J.A., 1994. Piezoelectric crystal for the detection of immunoreactions in buffer solutions. Sensors and Actuators B-Chemical 17(2), 125-131.

Guo, B., Guilford, W.H., 2004. The tail of myosin reduces actin filament velocity in the in vitro motility assay. Cell Motility and the Cytoskeleton 59(4), 264-272.

Hanson, K.L., Viidyanathan, V., Nicolau, D.V., 2006. Actomyosin motility detection using quartz crystal microbalance.

Harada, Y., 1990. Mechanochemical coupling in actomyosin energy transduction studied by in vitro movement assay. Journal of Molecular Biology 216, 49-68.

Harris, D.E., Warshaw, D.M., 1993. Smooth and Skeletal-Muscle Myosin Both Exhibit Low Duty Cycles at Zero Load in-Vitro. Journal of Biological Chemistry 268(20), 14764-14768.

Hiratsuka, Y., Tada, T., Oiwa, K., Kanayama, T., Uyeda, T.Q.P., 2001. Controlling the direction of kinesin-driven microtubule movements along microlithographic tracks. Biophysical Journal 81(3), 1555-1561.

Holzbaur, E.L., Goldman, Y.E., 2010. Coordination of molecular motors: from in vitro assays to intracellular dynamics. Current Opinion in Cell Biology 22(1), 4-13.

Hook, F., Kasemo, B., Nylander, T., Fant, C., Sott, K., Elwing, H., 2001. Variations in coupled water, viscoelastic properties, and film thickness of a Mefp-1 protein film during adsorption and cross-linking: A quartz crystal microbalance with dissipation monitoring, ellipsometry, and surface plasmon resonance study. Analytical Chemistry 73(24), 5796-5804.

Hook, F., Voros, J., Rodahl, M., Kurrat, R., Boni, P., Ramsden, J.J., Textor, M., Spencer, N.D., Tengvall, P., Gold, J., Kasemo, B., 2002. A comparative study of protein adsorption on titanium oxide surfaces using in situ ellipsometry, optical waveguide lightmode spectroscopy, and quartz crystal microbalance/dissipation. Colloids and Surfaces B-Biointerfaces 24(2), 155-170.

Huxley, A.F., Niedergerke, R., 1954. Structural changes in muscle during contraction Interference microscopy of living muscle fibres. Nature 173(4412), 971-973.

Huxley, H., Hanson, J., 1954. Changes in the cross-striations of muscle during contraction and stretch and their structural interpretation. Nature 173(4412), 973-976.

Jaber, J.A., Chase, P.B., Schlenoff, J.B., 2003. Actomyosin-driven motility on patterned polyelectrolyte mono- and multilayers. Nano Letters 3(11), 1505-1509.

Katira, P., Agarwal, A., Fischer, T., Chen, H.Y., Jiang, X., Lahann, J., Hess, H., 2007. Quantifying the performance of protein-resisting surfaces at ultra-low protein coverages using kinesin motor proteins as probes. Advanced Materials 19(20), 3171-3176.

Katira, P., Agarwal, A., Hess, H., 2009. A Random Sequential Adsorption Model for Protein Adsorption to Surfaces Functionalized with Poly(ethylene oxide). Advanced Materials 21(16), 1599-+.

Kinbara, K., Aida, T., 2005. Toward intelligent molecular machines: Directed motions of biological and artificial molecules and assemblies. Chemical Reviews 105(4), 1377-1400. 
Korten, S., Albet-Torres, N., Paderi, F., Ten Siethoff, L., Diez, S., Korten, T., Te Kronnie, G., Månsson, A., 2013. Sample solution constraints on motor-driven diagnostic nanodevices. Lab on a Chip - Miniaturisation for Chemistry and Biology 13(5), 866-876.

Korten, T., Månsson, A., Diez, S., 2010. Towards the application of cytoskeletal motor proteins in molecular detection and diagnostic devices. Current Opinion in Biotechnology 21(4), 477-488. Kron, S.J., Spudich, J.A., 1986. Fluoreescent actin filaments move on myosin fixated to a glass surface. Proceedings of the National Academy of Sciences of the United States of America 83(17), 6272-6276.

Kumar, S., Milani, G., Takatsuki, H., Lana, T., Persson, M., Frasson, C., Te Kronnie, G., Månsson, A., 2016. Sensing protein antigen and microvesicle analytes using high-capacity biopolymer nano-carriers. Analyst 141(3), 836-846.

Lard, M., ten Siethoff, L., Kumar, S., Persson, M., te Kronnie, G., Linke, H., Månsson, A., 2013. Ultrafast molecular motor driven nanoseparation and biosensing. Biosensors and Bioelectronics 48, 145-152.

Limberis, L., Stewart, R.J., 2000. Toward kinesin-powered microdevices. Nanotechnology 11(2), 47-51.

Mair, D.A., Geiger, E., Pisano, A.P., Fréchet, J.M.J., Svec, F., 2006. Injection molded microfluidic chips featuring integrated interconnects. Lab on a Chip - Miniaturisation for Chemistry and Biology 6(10), 1346-1354.

Margossian, S.S., Lowey, S., 1982. Preparation of mysoin and its subfragments from rabbit skeletal-muscle. Methods in Enzymology 85, 55-71.

Martinez-Neira, R., Kekic, M., Nicolau, D., dos Remedios, C.G., 2005. A novel biosensor for mercuric ions based on motor proteins. Biosensors \& Bioelectronics 20(7), 1428-1432.

Moorjani, S.G., Jia, L., Jackson, T.N., Hancock, W.O., 2003. Lithographically patterned channels spatially segregate kinesin motor activity and effectively guide microtubule movements. Nano Letters 3(5), 633-637.

Nicolau, D.V., Pham, D.K., Ivanova, E.P., Wright, J.P., Lenigk, R., Smekal, T., Grodzinski, P., 2005. Tone Reversal of an AFM Lateral Force Image Due to Hybridization of Oligonucleotides Immobilized on Polymers. Small 1(6), 610-613.

Nicolau, D.V., Solana, G., Kekic, M., Fulga, F., Mahanivong, C., Wright, J., dos Remedios, C.G., 2007a. Surface Hydrophobicity modulates the operation of actomyosin-based dynamic nanodevices. Langmuir 23, 10846-10854.

Nicolau, D.V., Solana, G., Kekic, M., Fulga, F., Mahanivong, C., Wright, J., Ivanova, E.P., dos Remedios, C.G., 2007b. Surface Hydrophobicity modulates the operation of actomyosin-based dynamic nanodevices. Langmuir 23, 10846-10854.

Nicolau, D.V., Suzuki, H., Mashiko, S., Taguchi, T., Yoshikawa, S., 1999. Actin motion on microlithographically functionalized myosin surfaces and tracks. Biophysical Journal 77(2), 1126-1134.

Nicolau, J., D. V., Lard, M., Korten, T., Van Delft, F.C.M.J.M., Persson, M., Bengtsson, E., Månsson, A., Diez, S., Linke, H., Nicolau, D.V., 2016. Parallel computation with molecularmotor-propelled agents in nanofabricated networks. Proceedings of the National Academy of Sciences of the United States of America 113(10), 2591-2596.

Nitta, T., Hess, H., 2005. Dispersion in Active Transport by Kinesin-Powered Molecular Shuttles. Nano Letters 5(7), 1337-1342.

Ostuni, E., Grzybowski, B.A., Mrksich, M., Roberts, C.S., Whitesides, G.M., 2003. Langmuir 19, 1861-1872. 
Rickert, J., Brecht, A., Gopel, W., 1997. Quartz crystal microbalances for quantitative biosensing and characterizing protein multilayers. Biosensors \& Bioelectronics 12(7), 567-575.

Riveline, D., Ott, A., Julicher, F., Winkelmann, D.A., Cardoso, O., Lacapere, J.J., Magnusdottir, S., Viovy, J.L., Gorre-Talini, L., Prost, J., 1998. Acting on actin: the electric motility assay. European Biophysics Journal with Biophysics Letters 27(4), 403-408.

Sauerbrey, G., 1959. Verwendung von schwingquartzen zur wagung dunner schichten und zur mikrowagung. Zeitschrift Fur Physik 155(2), 206-222.

Sellers, J.R., Cuda, G., Wang, F., Homsher, E., 1993. Myosin-specific adaptations of the motility assay. Methods in Cell Biology, Vol 39 39, 23-49.

Sollier, E., Murray, C., Maoddi, P., Di Carlo, D., 2011. Rapid prototyping polymers for microfluidic devices and high pressure injections. Lab on a Chip - Miniaturisation for Chemistry and Biology 11(22), 3752-3765.

Spudich, J.A., 2011. Molecular motors: Forty years of interdisciplinary research. Molecular Biology of the Cell 22(21), 3936-3939.

Spudich, J.A., Watt, S., 1971. Regulation of rabbit skeletal contraction 1. Biochemical studies on interaction of tropomyosin-troponin complex with actin and proteolytic fragments of myosin. Journal of Biological Chemistry 246(15), 4866-\&.

Sundberg, M., Balaz, M., Bunk, R., Rosengren-Holmberg, J.P., Montelius, L., Nicholls, I.A., Omling, P., Tagerud, S., Mansson, A., 2006. Selective spatial localization of actomyosin motor function by chemical surface patterning. Langmuir 22(17), 7302-7312.

Sundberg, M., Rosengren, J.P., Bunk, R., Lindahl, J., Nicholls, I.A., Tagerud, S., Omling, P., Montelius, L., Mansson, A., 2003. Silanized surfaces for in vitro studies of actomyosin function and nanotechnology applications. Analytical Biochemistry 323(1), 127-138.

Suzuki, H., Yamada, A., Oiwa, K., Nakayama, H., Mashiko, S., 1997. Control of actin moving trajectory by patterned poly(methyl methacrylate) tracks. Biophysical Journal 72(5), 1997-2001.

Taussky, H.H., Shorr, E., 1953. A microcolorimetric method for the determination of inorganic phosphorus. Journal of Biological Chemistry 202(2), 675-685.

Tawada, K., Sekimoto, K., 1991. A Physical Model of Atp-Induced Actin-Myosin Movement Invitro. Biophysical Journal 59(2), 343-356.

Toyoshima, Y.Y., Kron, S.J., McNally, E.M., Niebling, K.R., Toyoshima, C., Spudich, J.A., 1987. Myosin subfragment-1 is sufficient to move actin filaments in vitro. Nature 328(6130), 536-539.

Uyeda, T.Q.P., Kron, S.J., Spudich, J.A., 1990. Myosin Step Size - Estimation from Slow Sliding Movement of Actin over Low-Densities of Heavy-Meromyosin. Journal of Molecular Biology 214(3), 699-710.

Uyeda, T.Q.P., Warrick, H.M., Kron, S.J., Spudich, J.A., 1991. Quantized Velocities at Low Myosin Densities in an Invitro Motility Assay. Nature 352(6333), 307-311.

Vale, R.D., 2003. The molecular motor toolbox for intracellular transport. Cell 112(4), 467-480.

Van Zalinge, H., Aveyard, J., Hajne, J., Persson, M., Mansson, A., Nicolau, D.V., 2012. Actin filament motility induced variation of resonance frequency and rigidity of polymer surfaces studied by quartz crystal microbalance. Langmuir 28(42), 15033-15037.

Vasina, E.N., Paszek, E., Nicolau, D.V., 2009. The BAD project: data mining, database and prediction of protein adsorption on surfaces. Lab on a Chip 9(7), 891-900.

Veigel, C., Schmidt, C.F., 2011. Moving into the cell: Single-molecule studies of molecular motors in complex environments. Nature Reviews Molecular Cell Biology 12(3), 163-176. 
Warshaw, D.M., Desrosiers, J.M., Work, S.S., Trybus, K.M., 1990. Smooth-Muscle Myosin Cross-Bridge Interactions Modulate Actin Filament Sliding Velocity Invitro. Journal of Cell Biology 111(2), 453-463. 


\section{TABLES}

Table 1. Surfaces, total and active HMM surface density and actin filament motility parameters

\begin{tabular}{|c|c|c|c|c|c|c|}
\hline Surface & $\begin{array}{c}\text { Contact } \\
\text { Angle }\left(^{\circ}\right)\end{array}$ & $\begin{array}{c}\text { Total HMM } \\
\text { Density }{ }^{1} \\
\left(\text { molecules } \mu \mathrm{m}^{-2}\right)\end{array}$ & $\begin{array}{c}\text { Active HMM } \\
\text { Density }^{2} \\
\left(\text { molecules } \mu \mathrm{m}^{-2}\right)\end{array}$ & $\begin{array}{c}\text { [active]/ } \\
\text { [total] HMM }\end{array}$ & $\begin{array}{l}\text { Motile } \\
\text { Filaments } \\
\quad(\%)\end{array}$ & $\begin{array}{l}\text { Average } \\
\text { velocity } \\
\left(\mu \mathrm{m} \mathrm{s}^{-1}\right)\end{array}$ \\
\hline PMMA & $70 \pm 0.6$ & $7024 \pm 652$ & $2553 \pm 296$ & $0.36 \pm 0.07$ & 57 & $2.62 \pm 1.22$ \\
\hline $\mathrm{NC}$ & $74 \pm 0.5$ & $4294 \pm 645$ & $2928 \pm 1014$ & $0.68 \pm 0.16$ & 75 & $3.45 \pm 1.28$ \\
\hline PtBMA & $83 \pm 2.3$ & $11935 \pm 459$ & $2442 \pm 466$ & $0.20 \pm 0.03$ & 48 & $1.68 \pm 0.86$ \\
\hline PBMA & $90 \pm 1.4$ & $8773 \pm 55$ & $1312 \pm 208$ & $0.15 \pm 0.02$ & 55 & $2.20 \pm 1.06$ \\
\hline PS & $91 \pm 1.7$ & $9177 \pm 661$ & $1046 \pm 181$ & $0.11 \pm 0.03$ & 61 & $2.15 \pm 1.05$ \\
\hline $\mathrm{NC}^{3}$ & - & - & $2509 \pm 328$ & - & - & \\
\hline $\mathrm{NC}^{4}$ & - & - & 2400 & - & - & \\
\hline $\mathrm{TMCS}^{5}$ & $75.1 \pm 1.3$ & $\sim 20000$ & $5000-7000$ & $\sim 0.3$ & & \\
\hline
\end{tabular}

Notes

${ }^{1}$ Calculated from QCM measurements, corrected for polymer effects.

${ }^{2}$ Calculated from ATPase data.

${ }^{3}$ Data from (Guo and Guilford 2004).

${ }^{4}$ Data from (Uyeda et al. 1991)

${ }^{5}$ Data from(Albet-Torres et al. 2007a). Due to the large amount of water trapped in the HMM layer, which can be up to $10 \mathrm{x}$ the mass of the protein, the total number of HMM is a relative approximation. Additionally, TMCS is a very flat, very rigid surface, with low similarity with the polymer materials discussed here. 


\section{FIGURE CAPTIONS}

Figure 1. Chemical structures of surfaces and polymers tested for in vitro actomyosin motility support. A: nitrocellulose (NC); B: poly(styrene) (PS); C: poly(methyl methacrylate) (PMMA); D: poly(butyl methacrylate) (PBMA); G: poly(tert-butyl methacrylate) (PtBMA).

Figure 2. Amount of HMM molecules adsorbed on various polymers as a measure of their surface hydrophobicity (a, top); and versus their water intake (b, bottom).

Figure 3. Multi-layer model of the [solid substrate]-[stiff polymer]-[polymer gel]-[HMM layer].

Figure 4. Active HMM molecules adsorbed on various polymers, and the ratio of active/total HMM molecules, versus the respective surface hydrophobicities (a, top); and versus the water intake in the respective polymer (b, bottom).

Figure 5. Histograms of the frequency of co-occurrences of velocity, velocity variation; and deflection angle of the motility of actin filaments on various HMM-functionalised polymeric surfaces.

Figure 6. Relationship between the average velocity of the actin filaments and total number of HMM molecules (a, top); active number of HMM molecules (b, middle); and the ratio of active per total number of HMM (c, bottom). 


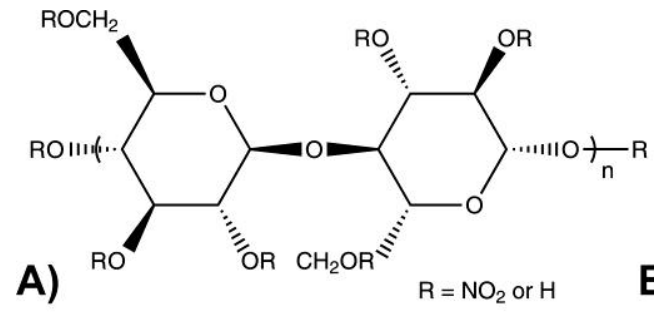

B)<smiles>CCC(CC)c1ccccc1</smiles><smiles>COC(=O)C1(C)C(C)C1(O)OC</smiles><smiles>CCOC(=O)C(C)(C(C)(C)C)C(C)(C)C</smiles>

C)

D)

E)

Figure 1. Chemical structures of surfaces and polymers tested for in vitro actomyosin motility support. A: nitrocellulose (NC); B: poly(styrene) (PS); C: poly(methyl methacrylate) (PMMA); D: poly(butyl methacrylate) (PBMA); G: poly(tert-butyl methacrylate) (PtBMA). 

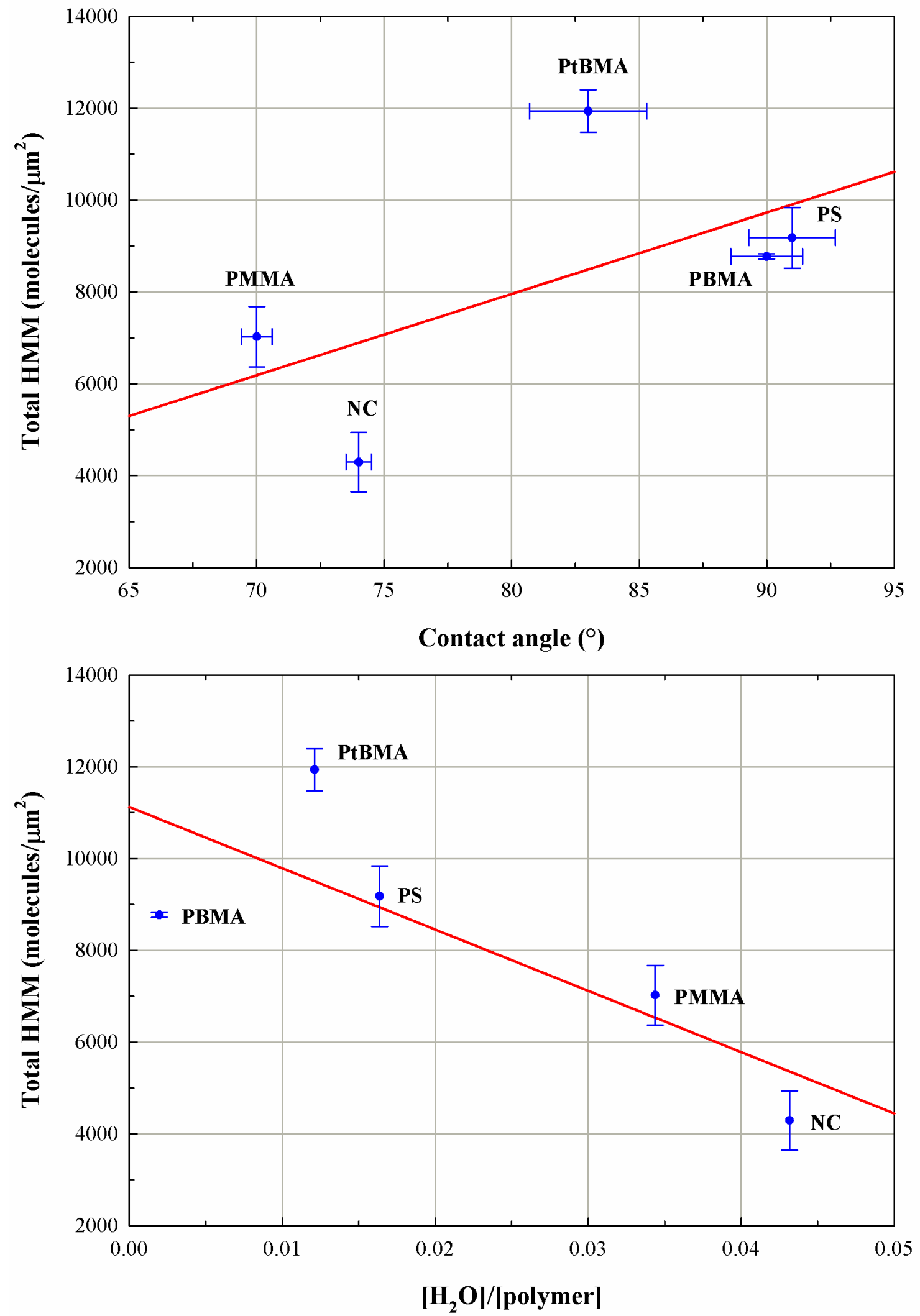

Figure 2. Amount of HMM molecules adsorbed on various polymers as a measure of their surface hydrophobicity (a, top); and versus their water intake (b, bottom). 


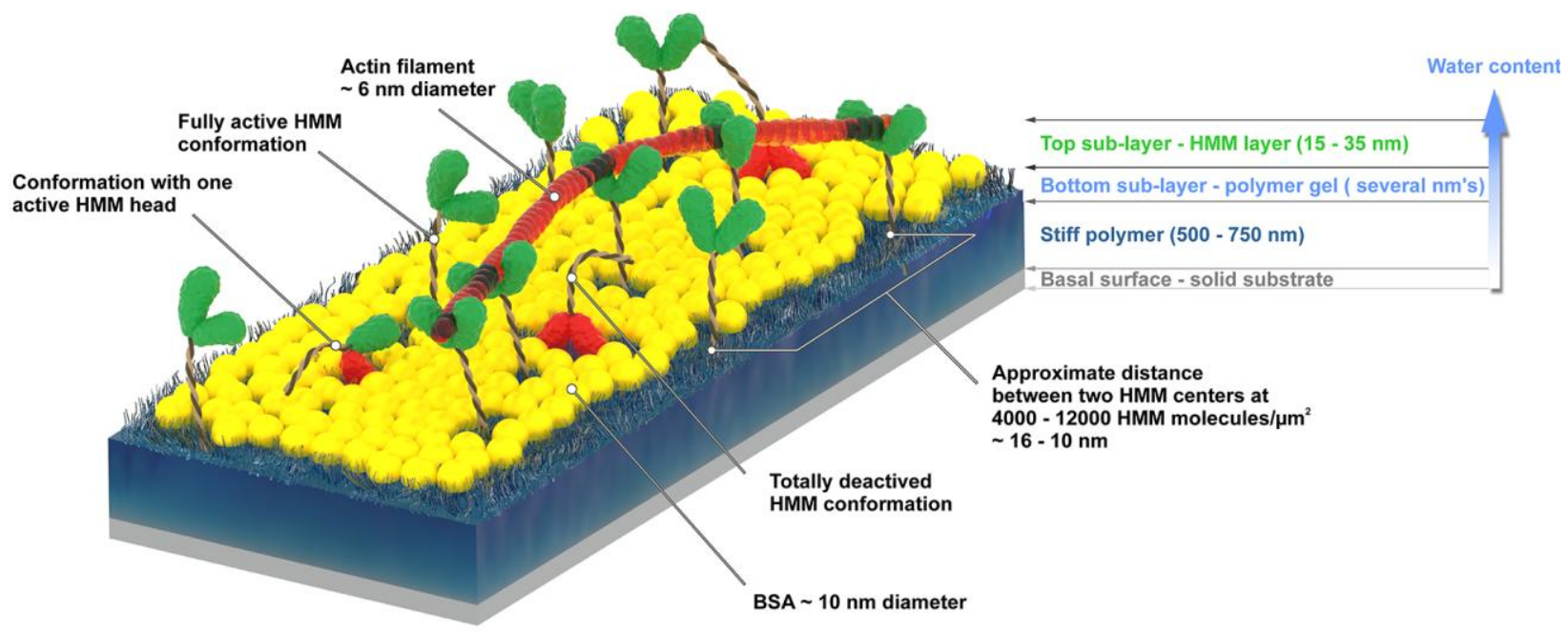

Figure 3. Multi-layer model of the [solid substrate]-[stiff polymer]-[polymer gel]-[HMM layer]. 

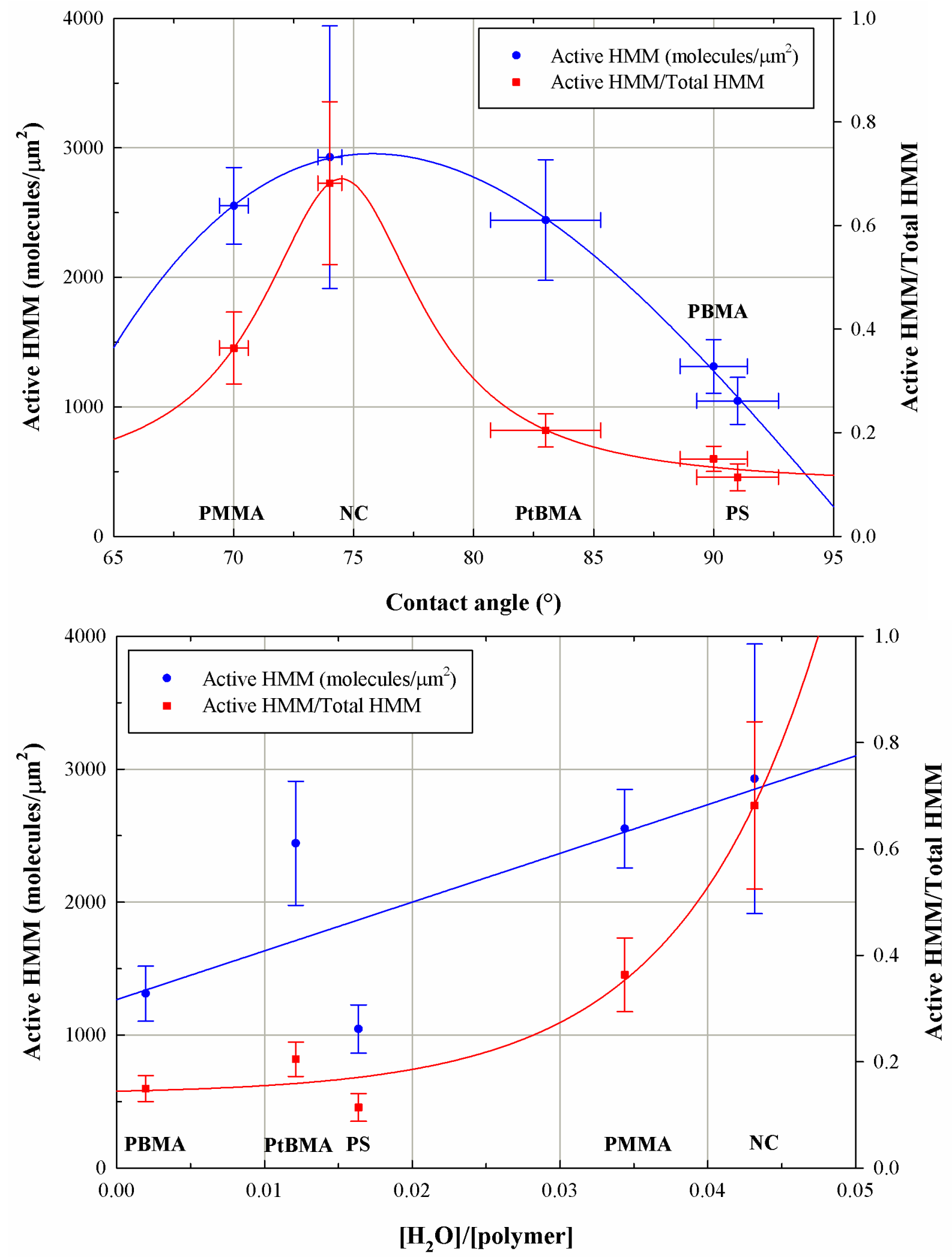

Figure 4. Active HMM molecules adsorbed on various polymers, and the ratio of active/total HMM molecules, versus the respective surface hydrophobicities (a, top); and versus the water intake in the respective polymer (b, bottom). 


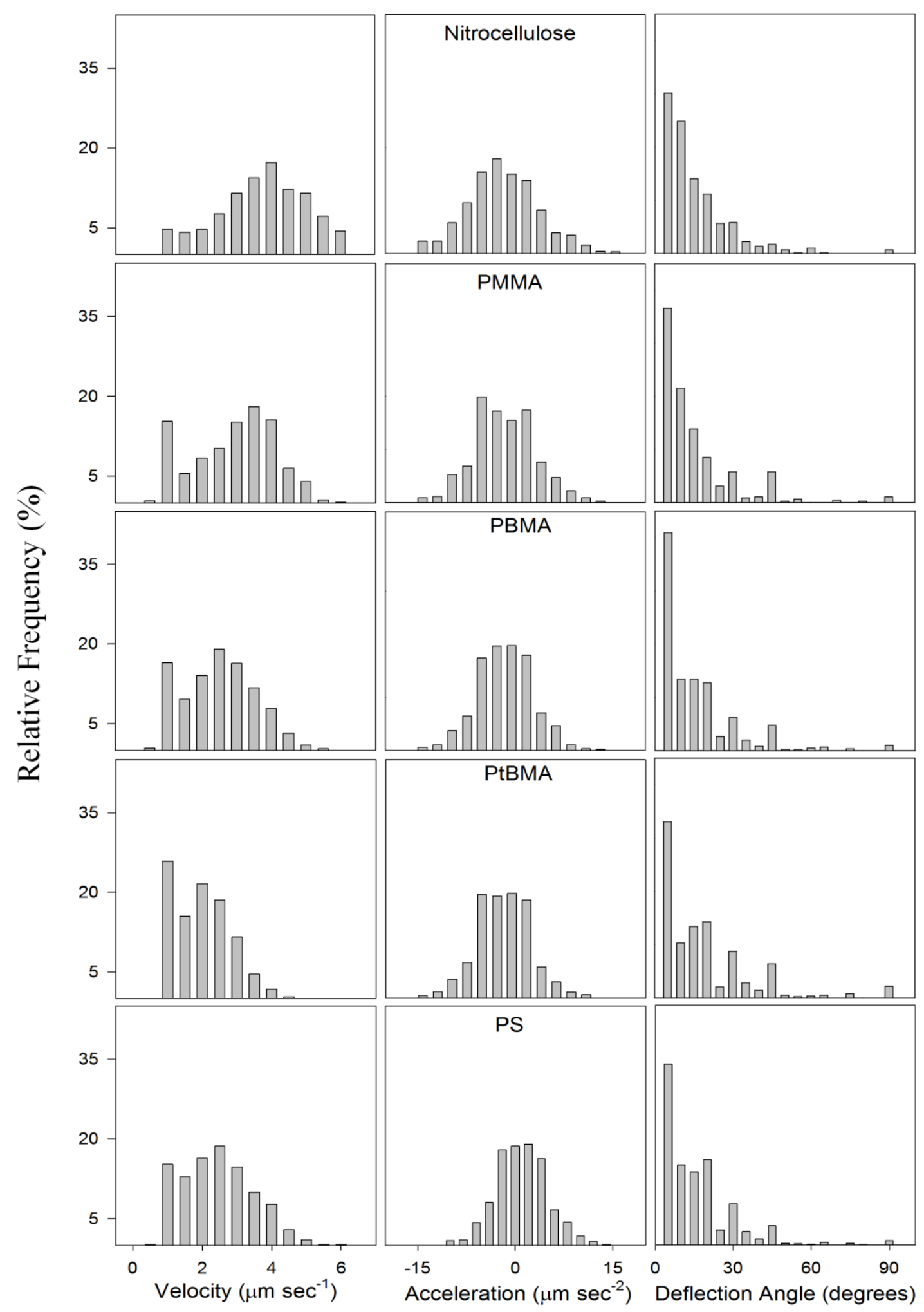

Figure 5. Histograms of the frequency of co-occurrences of velocity, velocity variation; and deflection angle of the motility of actin filaments on various HMM-functionalised polymeric surfaces. 


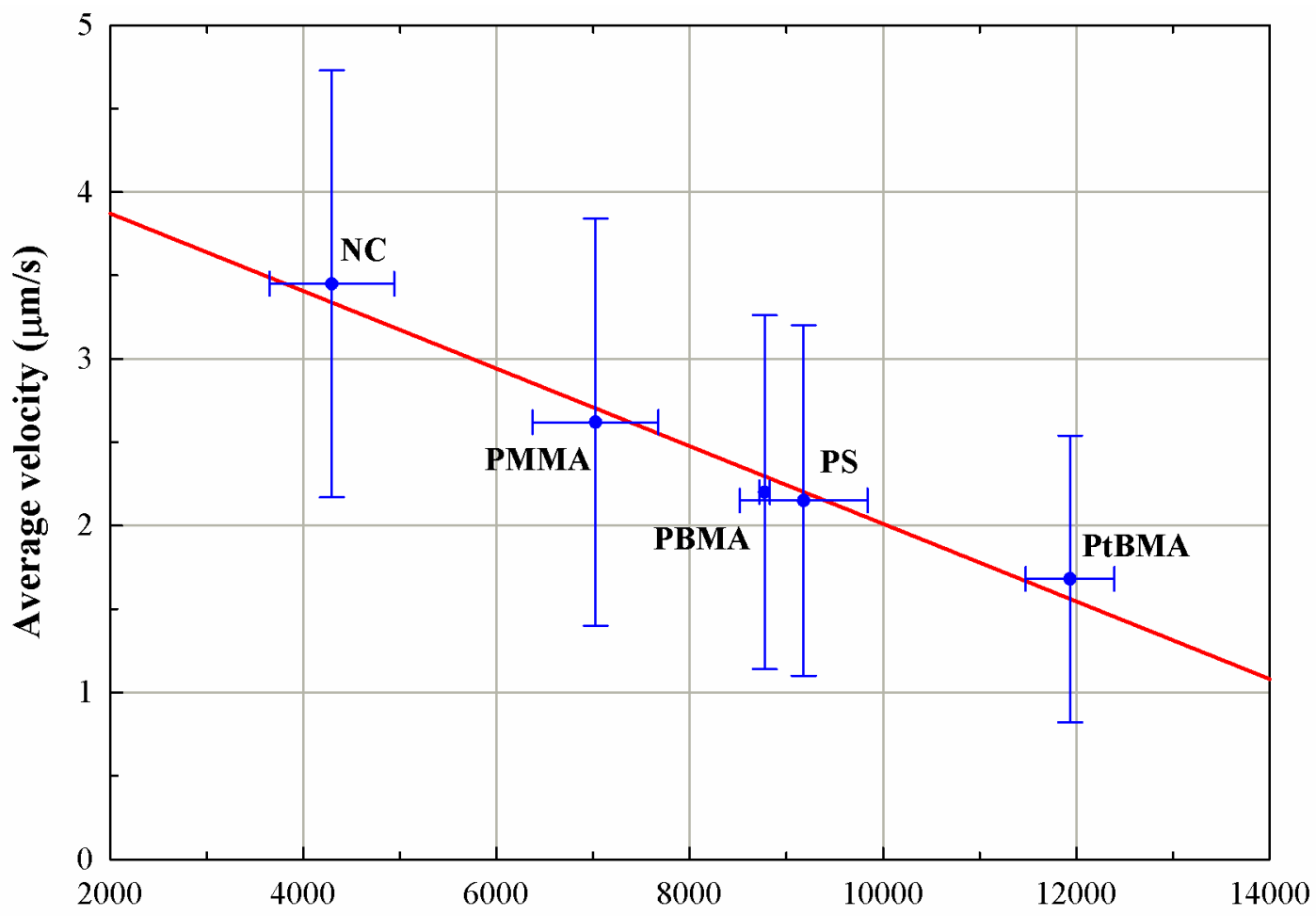

Total HMM (molecules $/ \mu^{2}$ )

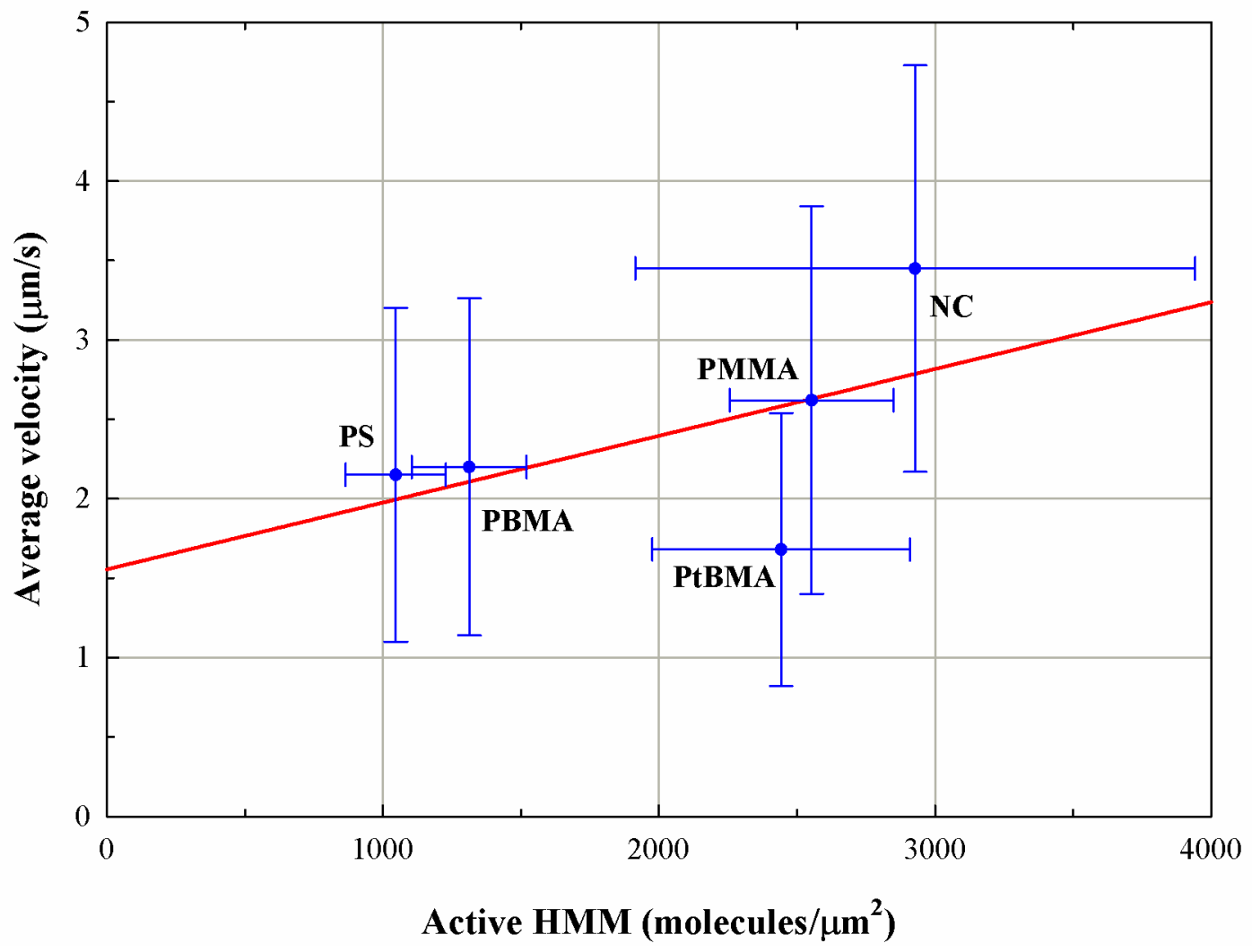




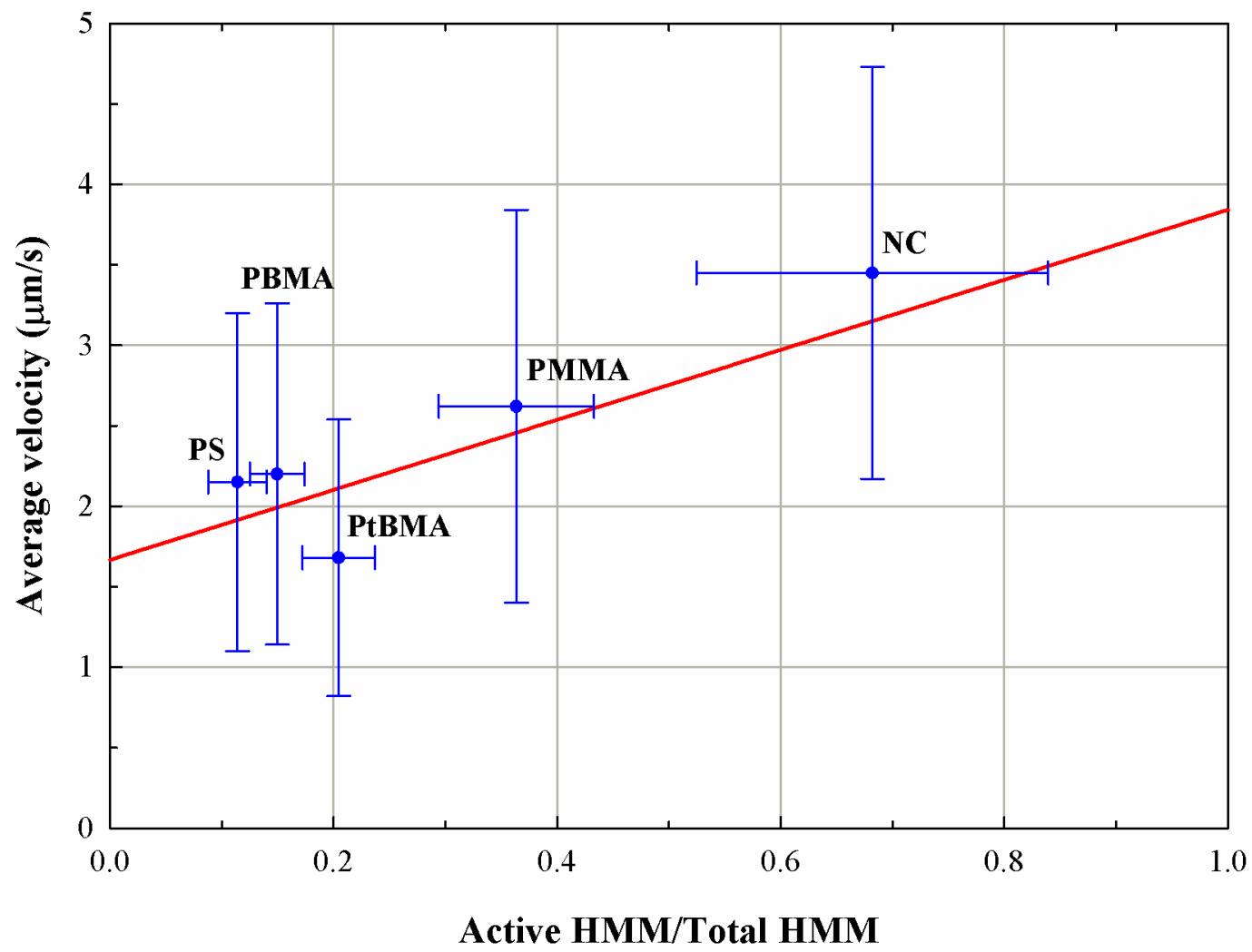

Figure 6. Relationship between the average velocity of the actin filaments and total number of HMM molecules (a, top); active number of HMM molecules ( $b$, middle); and the ratio of active per total number of HMM (c, bottom). 